\title{
A simple approach for accurate peptide quantification in MS- based proteomics
}

\author{
Teresa Mendes Maia ${ }^{\ddagger, 0, \|}$, An Staes ${ }^{\ddagger, 0, \|}$, Kim Plasman $^{\dagger}$, Jarne Pauwels $s^{\ddagger, 0, \|}$, Katie Boucher ${ }^{\ddagger, 0, \|}$, An- \\ drea Argentini $i^{\ddagger, \diamond, 1}$, Lennart Martens ${ }^{\ddagger, \diamond, i}$, Tony Montoye ${ }^{\ddagger}$, Kris Gevaert ${ }^{\ddagger, \diamond, \downarrow, *}$ \& Francis Impens \\ $\ddagger, 0, \|,+, *$
}

‡ VIB-UGent Center for Medical Biotechnology, Albert Baertsoenkaai 3, 90oo Ghent, Belgium

${ }^{\diamond}$ Department of Biomolecular Medicine, Ghent University, Albert Baertsoenkaai 3, 9ooo Ghent, Belgium

"I VIB Proteomics Core, Albert Baertsoenkaai 3, 9000 Ghent, Belgium

${ }^{\dagger}$ Alzheimer Research Foundation, Kalkhoevestraat 1, 879o Waregem, Belgium

' Bioinformatics Institute Ghent, Ghent University, Ghent, Belgium.

${ }^{*}$ Business Development Management, VIB, 9000 Ghent, Belgium

KEYWORDS: peptide quantitation, bottom-up proteomics, quality control, method optimization

\begin{abstract}
Despite its growing popularity and use, bottom-up proteomics remains a complex analytical methodology. Its general workflow consists of three main steps: sample preparation, liquid chromatography coupled to tandem mass spectrometry (LC-MS/MS) and computational data analysis. Quality assessment of the different steps and components of this workflow is instrumental to identify technical flaws and to avoid loss of precious measurement time and sample material. However, assessment of the extent of sample losses along the sample preparation protocol, in particular after proteolytic digestion, is not yet routinely implemented because of the lack of an accurate and straightforward method to quantify peptides. Here, we report on the use of a microfluidic UV/visible spectrophotometer to quantify MS-ready peptides directly in MS loading solvent, consuming only $2 \mu \mathrm{l}$ of sample. We determined the optimal peptide amount for LC-MS/MS analysis on a Q Exactive HF mass spectrometer using a dilution series of a commercial $\mathrm{K}_{5} 62$ cell digest. Careful evaluation of selected LC and MS parameters allowed us to define $3 \mu \mathrm{g}$ as an optimal peptide amount to be injected on this particular LC-MS/MS system. Finally, using tryptic digests from human HEK293T cells, we showed that injecting equal peptide amounts, rather than approximated ones, results into less variable LC-MS/MS and protein quantification data. The obtained quality improvement together with easy implementation of the approach makes it possible to routinely quantify MS-ready peptides as a next step in daily proteomics quality control.
\end{abstract}

\section{Introduction}

Mass spectrometry (MS)-based proteomics is a key technology in modern life sciences, with a wide scope of applications for protein research. Due to technological advances in liquid chromatography (LC) and MS instrumentation, current LC-MS/MS systems perform at high sensitivity, resolution and speed (Bache et al., 2018; D. Kelstrup et al., 2017; Espadas, Borràs, Chiva, \& Sabidó, 2017; Meier et al., 2018). These improvements, coupled to robust bioinformatic pipelines now allow routine in-depth proteome measurements at high confidence, explaining the success of the major proteomics applications today: quantitative measurements of proteomes by so-called shotgun proteomics, identification of protein interaction partners by affinity purification MS (AP-MS), and mapping of the some of the most common post-translational modification (PTM) sites.
LC-MS/MS systems are inherently prone to fluctuations in performance. Whether an ion source that gets transiently unstable in the course of an analytical run, a mass analyzer with a drift in accuracy, or a chromatographic column whose stationary phase progressively deteriorates, the different components of an LC-MS/MS system vary during operation time (Tabb, 2013). Therefore, continuous monitoring is required to diagnose technical flaws and schedule maintenance interventions. For a very long time however, guidelines and standardized methods to assess the quality of MS-based proteomics data were lacking. Over the last decade, great progress has been made in proteomics quality control. On the one hand, various reference sample formulations have been designed and are now commercialized as standards to carry out QC analysis (Bittremieux, Tabb, et al., 2017)(Tabb, 2013). On the other hand, a number of metrics to assess LC-MS/MS performance have been developed (Bittremieux, Walzer, et al., 
2017; Rudnick et al., 2010), in particular for standard workflows of bottom-up data-dependent analysis. Examples of QC metrics that inform about the liquid chromatography step are full-width at half-maximum (FWHM) of the eluting peptide peak and retention time drifts of eluting peptides, which monitor the integrity of the chromatographic column and the applied gradient. In turn, mass spectrometer QC can be done through examination of mass accuracy, dynamic range and ion injection times. Importantly, various software tools for MS-based proteomics quality control (QC) have been created (Bittremieux, Valkenborg, Martens, \& Laukens, 2017), which allow extraction and visualization of QC metrics from analytical runs performed on standard samples. One such example is the cloud-based quality control system QCloud, which allows following instrument performance over time on a user-friendly web interface (Chiva et al., 2018).

Of utmost importance for the success of a proteomic experiment is the QC assessment at the sample preparation level, where the major sources of variation arise from (1) artificial and thus unwanted in vitro protein/peptide modifications, (2) incomplete protein digestion, (3) protein contamination introduced by users or present in reagents and (4) sample losses during sample transfer, enrichment steps, elution and reconstitution. While the extent at which the first three types of variation compromise the quality of an analytical run can be quantified (provided that the correct identification search settings are used), no standardized QC procedures exist that account for analyte losses after proteolytic digestion. Indeed, while the extracted amount of protein material from cell or tissue samples is typically determined by standard assays immediately upon lysis and/or protein extraction, or prior to digestion (Figure 1), no such established quantification procedures exist at the peptide level. Of particular note, even when additional modifications, enrichment steps or cleaning procedures take place after digestion, which will inevitably introduce additional sample losses and variability, peptide quantification before the actual LC-MS/MS analysis is generally not performed.

LC-MS/MS runs carried out with insufficient amounts of peptides result in ion signals below the detection limit of the mass spectrometer, leading to low(er) numbers of peptide and protein identifications. In this respect, low signal to noise ratios of peptide signals also reduce the reliability of peptide quantification, as peak integration is more difficult for such low signals. Although analysis can be repeated by injecting more adequate sample amounts, this increases the overall analysis time and associated costs, and is not always possible for low quantity or low volume samples. Equally troublesome is the injection of excessive amounts of peptide material, termed column overloading. Such column saturation leads to peak tailing and retention time shifts (Johnson, Boyes, \& Orlando, 2013; McCalley, 2003, 2010; Rieux, Lubda, Niederländer, Verpoorte, \& Bischoff, 2006; Rieux, Sneekes, \& Swart, n.d.), leading to poorer pep-

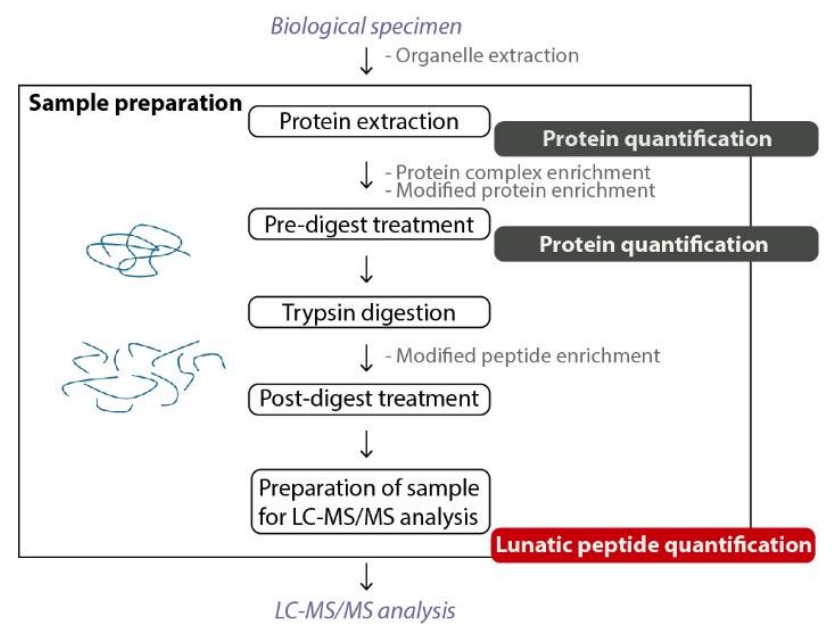

Figure 1 - Schematic outline of the sample preparation and quantification steps in a generic bottom-up proteomics experiment. Peptide concentration, which is usually estimated based on protein quantification before proteolytic digestion (dark gray), can be assessed right before LC-MS/MS using the Lunatic instrument (red). Optional steps of the protocol are written in gray.

tide separation and higher ionization competition, also reducing the number of identified and quantified peptides (Hinzke, Kouris, Hughes, Strous, \& Kleiner, 2019). In some cases, this saturation can even lead to obstruction of the column, which causes back-pressure build-up, in its turn increasing the variability between repeated analyses of similar samples.

Efforts to standardize procedures and minimize sample losses during sample preparation led to the development of simplified uniform protocols, including protocols in which all pre-digestion steps, being lysis, protein reduction and alkylation are performed in a single volume (Batth et al., 2019; Kulak, Pichler, Paron, Nagaraj, \& Mann, 2014; Moggridge, Sorensen, Morin, \& Hughes, 2018). Solid phase extraction (SPE) to purify and concentrate peptides has also become common practice at the end of the sample preparation protocol. Furthermore, automated sample preparation workflows (Fu et al., 2018; Guryča et al., 2014) or robotized systems start to become available, which allow accurate liquid handling, temperature control and precise timing of processing steps (Kulak, Geyer, \& Mann, 2017; Zhu et al., 2018). Some of these methods can be performed in miniaturized settings, which are advantageous when working with clinical or other low abundance samples where analyte amounts are scarce (Peng et al., 2016; Zhu et al., 2018).

Even when optimized sample preparation protocols are in place, straightforward QC assessment at the peptide level would be highly desirable for monitoring sample losses, leading to injecting optimal peptide amounts for LC-MS/MS analysis. In order for such a procedure to be aligned with a miniaturized, high-throughput mass spec- 
trometry workflow, it should allow (1) batch-wise processing, (2) low sample consumption and (3) execution just before LC-MS/MS analysis in the loading solvent used. Here, we devised a procedure that complies with these requirements, based on peptide quantification in a microfluidics UV spectrophotometer (Lunatic, Unchained Labs). Consuming only $2 \mu \mathrm{l}$ of sample, this instrument measures peptide concentrations as low as $31.25 \mathrm{ng} / \mu \mathrm{l}$ and returns the sample volume that should be injected for LC-MS/MS analysis of a desired peptide amount. Using this procedure, we determined the optimal amount of peptides to be injected for shotgun measurements on our LC-MS/MS setup. Finally, we demonstrated that the application of this procedure for control of sample loading improves LC-MS/MS and protein quantitation reproducibility.

\section{Methods}

\section{Sample preparation}

HEK293 cells $\left(5 \times 5 \times 10^{6}\right)$ were harvested, washed three times in PBS and resuspended in lysis buffer ( $8 \mathrm{M}$ urea; 20 mM HEPES pH 8.o). Samples were sonicated by 10 pulses of $30 \mathrm{~s}$ at an amplitude of $20 \%$, in a water bath kept at $10{ }^{\circ} \mathrm{C}$ and centrifuged for $15 \mathrm{~min}$ at $20,000 \mathrm{~g}$ at room temperature to remove insoluble components. The protein concentration in the supernatants of each sample was measured using a Bradford assay (Bio-Rad) and equal protein amounts (50o $\mu \mathrm{g}$ each) were used for further analysis. Proteins were reduced with $5 \mathrm{mM}$ DTT (Sigma-Aldrich) for $30 \mathrm{~min}$ at $55^{\circ} \mathrm{C}$. Alkylation was performed by addition of $10 \mathrm{mM}$ iodoacetamide (Sigma-Aldrich) for $15 \mathrm{~min}$ at room temperature in the dark. The samples were diluted with $20 \mathrm{mM}$ HEPES $\mathrm{pH} 8.0$ to a urea concentration of $4 \mathrm{M}$ and proteins were pre-digested with $5 \mu$ g endoLysC (Wako, 129-02541) $(\mathrm{i} / 100, \mathrm{w} / \mathrm{w})$ for $4 \mathrm{~h}$ at $37^{\circ} \mathrm{C}$. All samples were further diluted with $20 \mathrm{mM}$ HEPES $\mathrm{pH}$ 8.o to a final urea concentration of $2 \mathrm{M}$ and proteins were digested with $5 \mu \mathrm{g}$ trypsin (V5111, Promega) (1/100, w/w) overnight at $37{ }^{\circ} \mathrm{C}$. Peptides were then purified on a SampliQ SPE C18 cartridge (Agilent), vacuum dried and kept at $-20{ }^{\circ} \mathrm{C}$ until further use.

\section{Peptide concentration measurements}

All peptide concentration measurements were made on the Lunatic (Unchained labs) microfluidic spectrophotometer. Quantification of peptide mixtures was determined by the instrument's "MS-peptide quant" tool, a software application that computes peptide concentration values based on UV-absorbance (280 $\mathrm{nm}$ ) of aromatic amino acids.

Linear dynamic range determination
The linear dynamic range for measurement of complex peptide mixtures on the Lunatic was determined on a group of eight solutions prepared from a $1.0 \mu \mathrm{g} / \mu \mathrm{l}$ stock of a commercial yeast protein digest (Promega, V7461), dissolved in loading solvent (o.1\% TFA in acetonitrile/water, $2 / 98(\mathrm{v} / \mathrm{v}))$. Measurements of solutions in the $1.0 \mu \mathrm{g} / \mu \mathrm{l}$ to $10 \mathrm{ng} / \mu \mathrm{l}$ concentration range (used concentrations were 0.010, 0.025, 0.050, 0.100, 0.250, 0.500, 0.750, $1.000 \mu \mathrm{g} / \mu \mathrm{l})$ were done in quadruplicate, from four yeast protein digest vials, and at least 2 readouts per solution were taken.

\section{Assessment of optimal loading amount}

A commercially available $\mathrm{K}_{5} 62$ cell digest (Promega, V6951) was used to create a two-fold dilution series ranging from 0.013 to $1.0 \mu \mathrm{g} / \mu \mathrm{l}$. Peptide amounts ranging from 0.06 $\mu \mathrm{g}$ up to $12 \mu \mathrm{g}$ were loaded on a LC-MS/MS system. In parallel, the actual sample concentration values were assessed by Lunatic (see Figure 3a). The same experiment was repeated using a new $\mathrm{K}_{5} 6_{2}$ cell vial to prepare the dilution series and a different analytical column.

Assessment of LC-MS/MS analysis quality under controlled sample loading conditions

Five HEK293 protein digest samples were resuspended in $30 \mu \mathrm{l}$ MS loading solvent (o.1\% TFA in acetonitrile/water, 2/98 (v/v)) and measured on Lunatic. Two sets of LCMS/MS analytical runs followed. In the first one, $3.0 \mu \mathrm{g}$ of peptide mixture from each sample was loaded onto the LCMS/MS system, while in the second one the same sample volume was used for all five injections, resulting in peptide amounts ranging from 2.50 to $3.55 \mu \mathrm{g}$ (detailed in the Results section).

\section{LC-MS/MS analysis}

From each sample, $5 \mu \mathrm{l}$ was introduced into an LC-MS/MS system through an Ultimate 3000 RSLC nano LC (Thermo Fisher Scientific, Bremen, Germany) in-line connected to a Q Exactive HF mass spectrometer (Thermo Fisher Scientific). The sample mixture was first loaded on a trapping column (made in-house, $100 \mu \mathrm{m}$ internal diameter (I.D.) $\times 20 \mathrm{~mm}, 5 \mu \mathrm{m}$ beads $\mathrm{C}_{1} 8$ Reprosil-HD, Dr. Maisch, Ammerbuch-Entringen, Germany). After flushing from the trapping column, the sample was loaded on an analytical column (made in-house, $75 \mu \mathrm{m}$ I.D. $\times 400 \mathrm{~mm}$, $1.9 \mu \mathrm{m}$ beads C18 Reprosil-HD, Dr. Maisch) packed in the needle (pulled in-house). Peptides were loaded with loading solvent (o.1\% TFA in acetonitrile/water, $2 / 98(\mathrm{v} / \mathrm{v}))$ and eluted with a non-linear 150 min gradient of $2-56 \%$ solvent B (o.1\% formic acid in water/acetonitrile, $20 / 80(\mathrm{v} / \mathrm{v}))$ at a flow rate of $250 \mathrm{~nL} / \mathrm{min}$. This was followed by a $15 \mathrm{~min}$ wash reaching $99 \%$ solvent $\mathrm{B}$ and re-equilibration with solvent 
A (o.1\% formic acid). The column temperature was kept constant at $50{ }^{\circ} \mathrm{C}$ (CoControl 3.3.05, Sonation).

The mass spectrometer was operated in data-dependent, positive ionization mode, automatically switching between MS and MS/MS acquisition for the 16 most abundant peaks in a given MS spectrum. The source voltage was set to 3.0 $\mathrm{kV}$ and the capillary temperature was $250^{\circ} \mathrm{C}$. Full scan MS spectra $\left(375^{-1,500} \mathrm{~m} / \mathrm{z}\right.$, AGC target $3 \times 10^{6}$ ions, maximum ion injection time of $45 \mathrm{~ms}$ ) were acquired at a resolution of $60,000($ at $200 \mathrm{~m} / \mathrm{z})$ in the orbitrap analyzer, followed by up to 16 tandem MS scans (resolution 15,000 at $200 \mathrm{~m} / \mathrm{z}$ ) of the most intense ions fulfilling predefined selection criteria (AGC target $1 \times 10^{5}$ ions, maximum ion injection time of $60 \mathrm{~ms}$, isolation window of $1.5 \mathrm{~m} / \mathrm{z}$, fixed first mass of 145 $\mathrm{m} / \mathrm{z}$, spectrum data type: centroid, underfill ratio $2 \%$, intensity threshold $1.3 \times 10^{4}$, exclusion of unassigned, singly and $>7$ charged precursors, peptide match preferred, exclude isotopes on, dynamic exclusion time of $12 \mathrm{~s}$ ). The HCD collision energy was set to $28 \%$ of the normalized collision energy and the polydimethylcyclosiloxane background ion at 445.12002 Da was used for internal calibration (lock mass).

\section{Data analysis}

Data analysis was performed with the MaxQuant software (version 1.6.1.o) (Cox \& Mann, 2008). The Andromeda search engine was used with default settings, including PSM, peptide and protein false discovery rate set at $1 \%$ and match between runs disabled. Spectra were searched against the UniProt reference proteome release 2018_01 (UPooooo5640_9606, containing 21007 human protein entries) with a mass tolerance for precursor and fragment ions of 4.5 and $20 \mathrm{ppm}$, respectively. Oxidation of methionine residues and acetylation of protein $\mathrm{N}$-termini were defined as variable modifications, while carbamidomethylation of cysteine residues was set as a fixed modification. Proteins with at least one unique or razor peptide were retained, then quantified by the MaxLFQ algorithm integrated in the MaxQuant software (Cox et al., 2014). A minimum ratio count of two unique or razor peptides was required for quantification. Further data analysis was performed with the Perseus software (version 1.6.1.3) after loading the protein groups file from MaxQuant. The number of protein identifications was given by the number of protein groups obtained after filtering out protein groups only identified by site, reverse database hits and potential contaminants. The number of peptide identifications was obtained after exclusion of reverse database hits and potential contaminants. Quantified proteins were the subset of identified proteins with positive LFQ intensities. Heat maps were prepared from matrices of $\log _{2}$ transformed LFQ protein expression values, after filtering for at least three valid values over all samples. Proteins were displayed according to the order of groups defined by hierarchical clustering, using Euclidean distances and average linkage settings.

The chromatographic parameters signal to noise $(\mathrm{S} / \mathrm{N})$ ratio and peak area were obtained from the moFF algorithm (Argentini et al., 2016), run on each analytical run. Peak areas were approximated by the area of a triangle of base equal to the peak width (in minutes) and height given by the peak's apex intensity. MS2 injection time was retrieved from MaxQuant's MS/MS scans table. This analysis was done on peptide-to-spectrum matches from features detected in every analytical run from each experiment. Peptides were partitioned according to hydrophobicity the following way: hydrophilic peptides were those eluting until 50 min of the LC gradient (1.6-12.3\% acetonitrile), intermediate hydrophobic peptides eluted between 50 and 110 min (12.3-26.6\% acetonitrile) and hydrophobic peptides eluted after $110 \mathrm{~min}$ (26.6-79.2 \% acetonitrile) (Figure $\mathrm{S}_{1}$ ).

The mass spectrometry proteomics data have been deposited to the ProteomeXchange Consortium (http://proteomecentral.proteomexchange.org) via the PRIDE partner repository with the identifier 10.6019/PXDo14524 (username: reviewer67074@ebi.ac.uk; password: 2RDeDBQ4).

\section{Results and Discussion}

The introduction of a peptide purification step prior to LC-MS/MS analysis (Peng et al., 2016) in routine in proteomics laboratories opened an opportunity to accurately determine peptide concentrations. Such a peptide purification step, typically based on Solid Phase Extraction (SPE), removes buffer components (like chaotropes, certain detergents, reducing/alkylating reagents and trypsin) that interfere with LC-MS/MS analysis. As these components often absorb UV light, for long time they prevented routine, fast and accurate peptide quantification, often leading to the analysis of suboptimal peptide amounts by LC-MS/MS. The high purity of peptides obtained after SPE led us to explore the possibility to quantify MS-ready peptide samples using a Lunatic spectrophotometer from Unchained Labs.

A simple application for accurate quantification of MSready peptide samples

The Lunatic instrument is a microfluidic spectrophotometer that performs DNA, RNA, protein and peptide quantifications in batch, consuming only $2 \mu \mathrm{l}$ of sample. Determination of peptide concentrations occurs accurately by a proprietary software application that, from an acquired UV-visible absorbance spectrum, discriminates the signal coming from the UV light absorbing amino acids from those of usual contaminants (e.g. nucleic acids, cofactors). The "MS-peptide Quant" application, available since September 2017 on Lunatic instruments, quantifies peptides by 
taking into account the extinction coefficients of tryptophan and tyrosine at $280 \mathrm{~nm}$, as well as estimated values for both their fractions in a protein digest and their proportion in mass relative to the whole protein content of a sample.

The ability to quantify complex peptide mixtures was verified using dilution series of commercial protein digests from yeast, ranging in concentration between $10 \mathrm{ng} / \mu \mathrm{l}$ and $1 \mu \mathrm{g} / \mu \mathrm{l}$, based on the theoretical peptide quantity as reported by the vendor. Comparison between the theoretical concentration values and the measured ones in four independent replicate dilutions showed a high linear correlation $\left(\mathrm{R}_{2}>0.98\right)$ and a slope very close to 1 (t-test $\mathrm{p}$-value $=$ o.19), demonstrating the instrument's high accuracy (Figure 2). It must be noted that peptide solutions with concentrations below $50 \mathrm{ng} / \mu \mathrm{l}$ gave absorbance measurements lower than 0.03 , the lower absorbance limit at which analyte concentration can be accurately assessed according to the Lunatic specifications. Excluding measurements from such samples, the coefficient of determination $\left(R^{2}\right)$ between the theoretical and observed peptide concentrations was above 0.98. Therefore, it was concluded that solutions of peptides with concentrations between $50 \mathrm{ng} / \mu \mathrm{l}$ and 1 $\mu \mathrm{g} / \mu \mathrm{l}$ are within the dynamic range of the instrument and can thus be accurately measured. This concentration range is well suited for MS-ready samples, for which LC-MS/MS experiments typically consume peptide quantities between $100 \mathrm{ng}$ and $5 \mu \mathrm{g}$, contained in a volume of a few $\mu$ l.

In summary, together with its accuracy for measurement of peptide solutions at commonly used concentration ranges, peptide quantitation with the Lunatic instrument presents a unique set of characteristics that make it particularly attractive over other quantification methods for MSready bottom-up proteomics samples (e.g. Bradford, Lowry, BCA, Pierce $660 \mathrm{~nm}$, tryptophan fluorescence (Wiśniewski \& Gaugaz, 2015)). It is a quick and easy method that can be performed in batch. It only implies a very low, $2 \mu \mathrm{l}$, sample consumption. It is based on UV-light absorbance at $280 \mathrm{~nm}$, an intrinsic property of aromatic amino acids, thus requiring no calibration curve, unlike colorimetric reaction-based assays like Bradford or BCA.

Determination of an optimal peptide amount for LCMS/MS analysis

The ideal peptide amount to use for LC-MS/MS in order to have a maximum throughput and identification rate, while keeping a good analyte separation, will largely depend on the characteristics of the LC-MS/MS system, namely on its chromatographic column loading capacity. If the exact sample concentration would be known, it would be possible to always work with optimal sample amounts, and thus consistently get high quality LC-MS/MS runs. Therefore, taking advantage of peptide quantification by the Lunatic instrument, we set out to determine the most suitable sample loading for an LC-MS/MS setup routinely
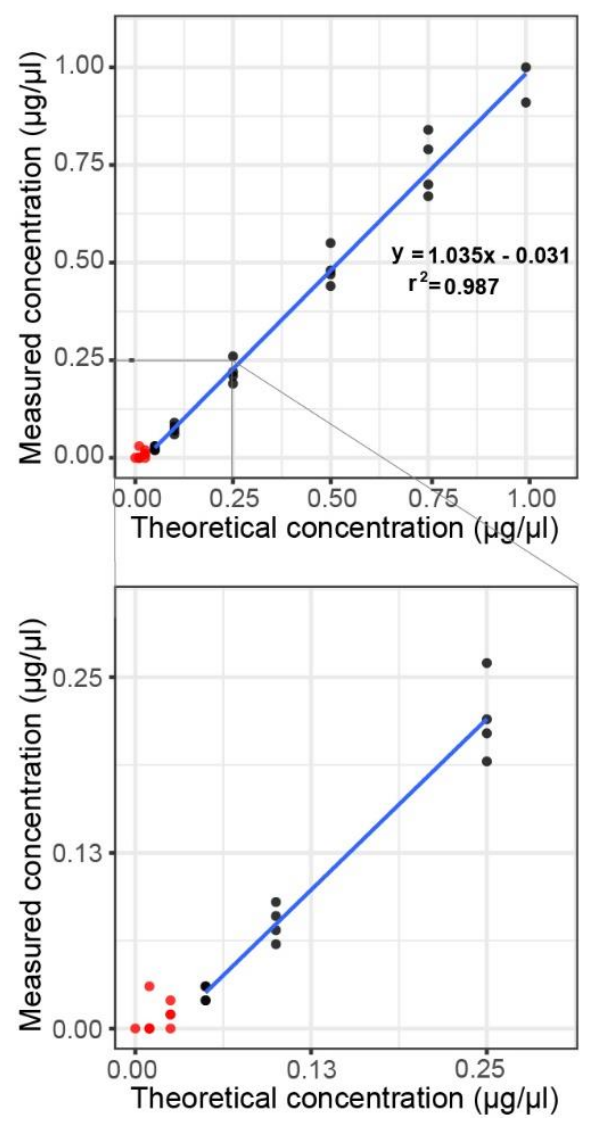

Figure 2 - Dynamic quantification range of the Lunatic spectrophotometer for complex peptide mixtures. Yeast peptide digest solutions with concentrations ranging from $10 \mathrm{ng} / \mu \mathrm{l}$ to $1.0 \mu \mathrm{g} / \mu \mathrm{l}$ (black dots) can be accurately measured on the Lunatic, as shown by a strong correlation between the theoretical and measured peptide concentration values. Below 50 $\mathrm{ng} / \mu \mathrm{l}$ (red dots), peptide measurements are no longer reliable, as absorbance values reach the lower limit of detection of the spectrophotometer. Dots represent measurements of four independent replicate dilutions.

used in our laboratory: a $40 \mathrm{~cm}$ LC column packed in the needle with $1.9 \mu \mathrm{m} \mathrm{C} 18$ beads coupled to a Q Exactive HF mass spectrometer.

Starting from a commercial tryptic digest of a $\mathrm{K}_{5} 62$ cell lysate, we prepared 10 different samples with peptide concentrations ranging from $0.013 \mu \mathrm{g} / \mu \mathrm{l}$ to $2.4 \mu \mathrm{g} / \mu \mathrm{l}$. Five microliter of these samples, containing peptide amounts ranging from 0.06 to $12 \mu \mathrm{g}$, was injected for LC-MS/MS analysis using a 2.5 hour gradient. The experiment was repeated to generate data for two independent series of replicates analyzed on two different LC columns (Figure 3, Figure S2).

All peptide concentration measurements were lower than predicted (Figure 3a). Likely, these deviations arose mostly from peptide losses during peptide resuspension of the $\mathrm{K}_{562}$ digest. Additional losses during the preparation of serially diluted samples were minor, judging from the ratios between consecutive dilutions, which were very 
bioRxiv preprint doi: https://doi.org/10.1101/703397; this version posted July 16,2019 . The copyright holder for this preprint (which was not certified by peer review) is the author/funder, who has granted bioRxiv a license to display the preprint in perpetuity. It is made available under aCC-BY-NC-ND 4.0 International license.

\begin{tabular}{|c|c|c|c|c|c|c|c|c|c|c|}
\hline \# Dilution & 1 & 2 & 3 & 4 & 5 & 6 & 7 & 8 & 9 & 10 \\
\hline Theoretical $(\mu g)$ & 0.06 & 0.13 & 0.25 & 0.50 & 1.00 & 2.00 & 4.00 & 6.00 & 8.00 & 12.00 \\
\hline LC column $1(\mu g)$ & $-0.08^{*}$ & $-0.07^{*}$ & 0.05 & 0.30 & 0.65 & 1.56 & 3.36 & 5.08 & 6.90 & 11.13 \\
\hline LC column $2(\mu g)$ & $-0.05^{*}$ & 0.03 * & 0.08 & 0.26 & 0.76 & 1.41 & 3.20 & 5.32 & 7.26 & 10.85 \\
\hline $\begin{array}{l}\text { Average }(\mu g) \\
(L C \text { column1 and } 2)\end{array}$ & $\underline{-0.07}$ & $\underline{-0.02}$ & $\underline{0.07}$ & $\underline{0.28}$ & $\underline{0.71}$ & $\underline{1.49}$ & $\underline{3.28}$ & $\underline{5.20}$ & $\underline{7.08}$ & $\underline{10.99}$ \\
\hline
\end{tabular}

*peptide measurements done below Lunatic spectrophotometer's detection limit

b

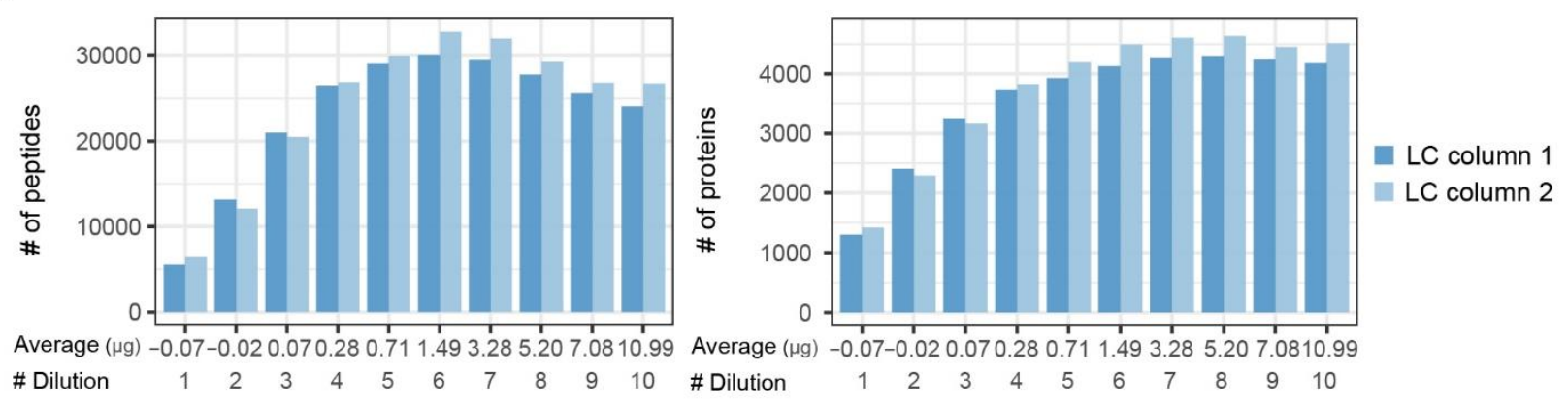

C

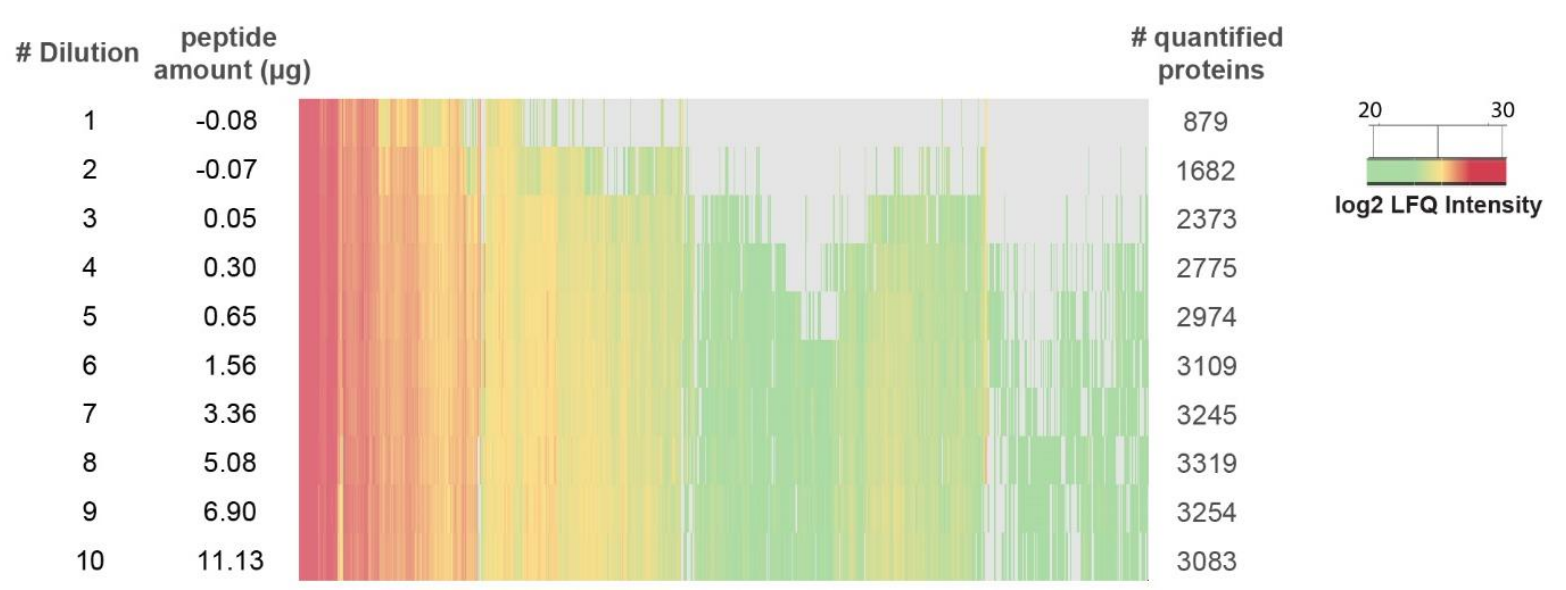

Figure 3 - Identified and quantified peptide and protein numbers upon LC-MS/MS analysis of 1o different amounts of a trypsin digested $\mathrm{K}_{5} 62$ cell extract. (a) Theoretical and measured peptide amounts for two independent experiments. In both experiments, all measured values were lower than predicted, pointing to peptide losses during sample solution preparation. (b) Bar plots indicating the numbers of identified peptide and proteins per analytical run. Colors represent the results of two replicate series analyzed on two different LC columns. (c) Representative heatmap visualizing the intensities of quantified proteins in each of the 10 analytical runs measured using LC column 1. Missing values are shown in gray. Based on numbers of identified and quantified proteins a rather broad window ranging from 1.49 to $5.20 \mu \mathrm{g}$ of peptides was found as an optimal injection amount.

close to expected $\left(\mathrm{R}^{2}>0.99\right)$. We also noticed that peptide quantification of \# Dilution 1 and 2, having theoretical concentrations of 0.013 and $0.025 \mu \mathrm{g} / \mu \mathrm{l}$, returned negative values. These clearly inaccurate measurements were performed below the limit of detection of the spectrophotometer.

To find an optimal peptide amount to inject on our LCMS/MS setup, we evaluated the number of identified and quantified proteins. To this end, the LC-MS/MS data were searched with MaxQuant (Cox \& Mann, 2008) and identified proteins were quantified by the MaxLFQ algorithm (Cox et al., 2014) (Figure 3b-c, Figure S2, Table S1). We observed that the number of identified proteins increased with the injected peptide amount until it started to level off at an average amount of 3.28 to $5.20 \mu \mathrm{g}$ on injected peptides. As for the numbers of identified peptides, these reached a maximum between 1.49 to $3.28 \mu \mathrm{g}$. This suggested that column overloading occurred for peptide amounts above $3.28 \mu \mathrm{g}$, leading to a marked decrease in the number of identified peptides (18 to $20 \%$ reduction in numbers of identified peptides for the $10.99 \mu \mathrm{g}$ samples). Although these peptide losses did not readily translate into a lower number of identified proteins, they did have an impact on the number of quantified proteins. Indeed, injection of an average amount of 3.28 to $5.20 \mu \mathrm{g}$ of peptides 
bioRxiv preprint doi: https://doi.org/10.1101/703397; this version posted July 16,2019 . The copyright holder for this preprint (which was not certified by peer review) is the author/funder, who has granted bioRxiv a license to display the preprint in perpetuity. It is made available under aCC-BY-NC-ND 4.0 International license.

a
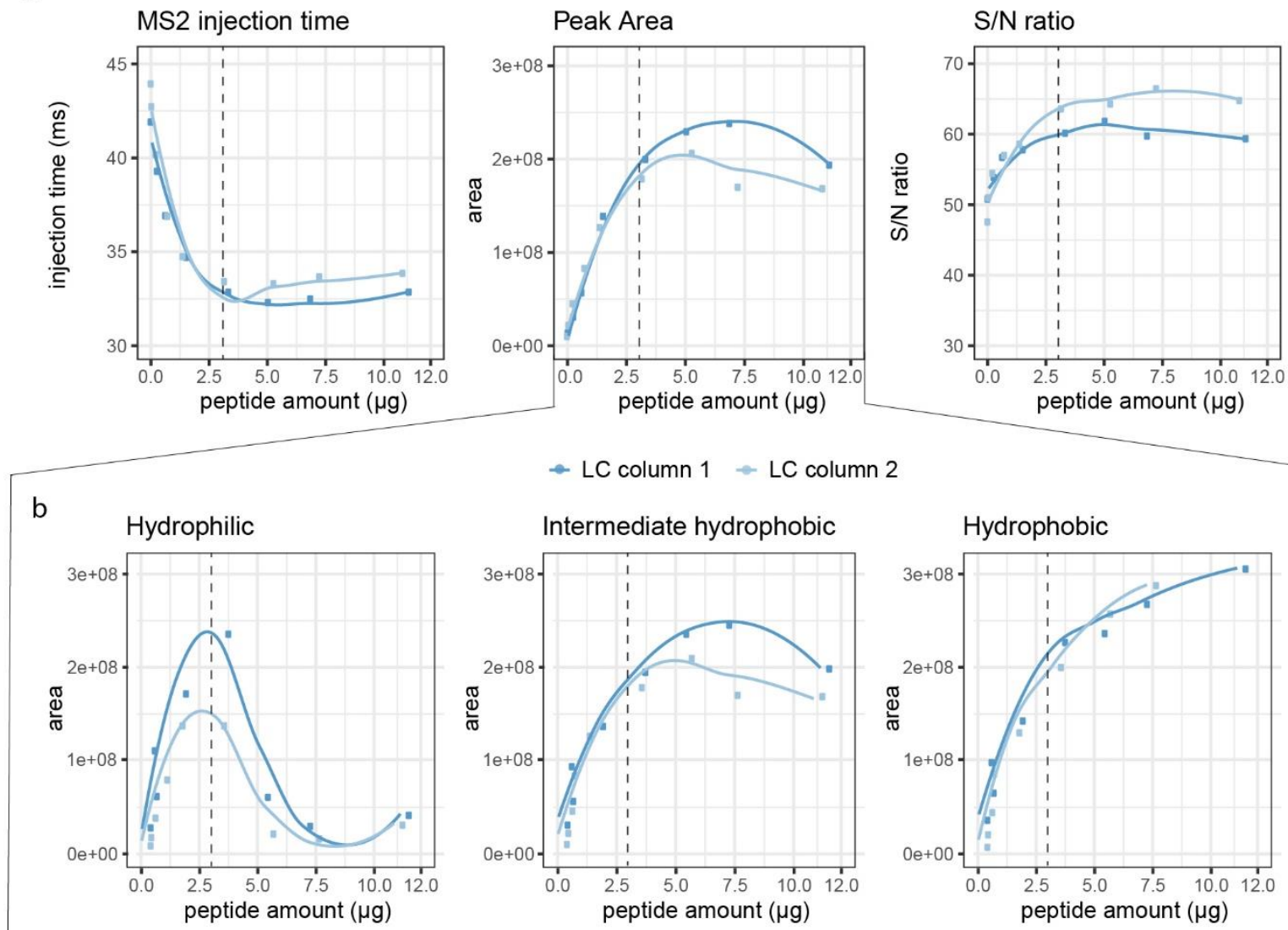

- LC column $1-$ LC column 2
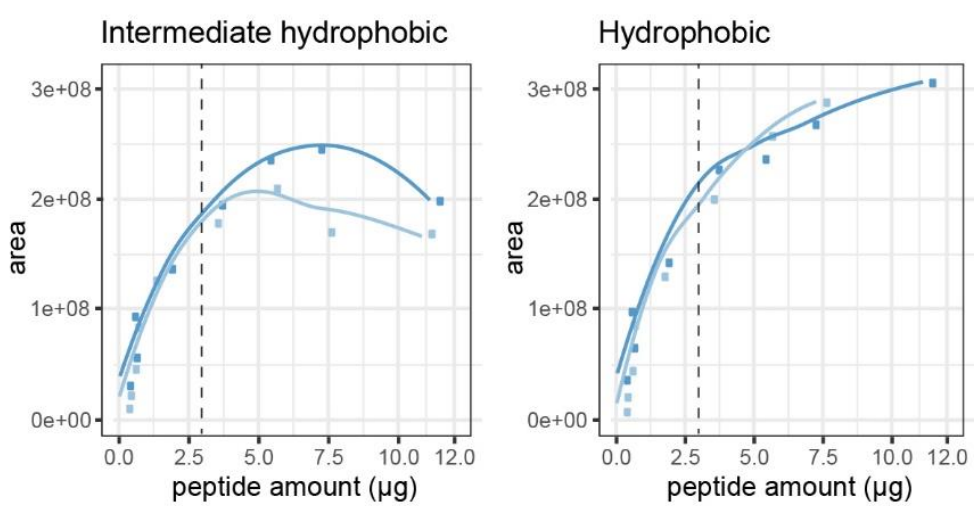

Figure 4 - Liquid chromatography and mass spectrometry metrics upon LC-MS/MS analysis of 10 different amounts of a trypsin digested $\mathrm{K}_{562}$ cell extract. (a) Scatter plots of average MS2 ion injection time, median peak area and median signal-to-noise ratio of identified peptides in each analytical run. (b) Scatter plots of the median peak area of hydrophilic, intermediate hydrophobic and hydrophobic identified peptides. Colors represent the results of two replicate series analyzed on two different LC columns. Loess curve fitting was used to generate the connecting lines. Based on the loss of hydrophilic peptides $3 \mu \mathrm{g}$ was defined as optimal peptide injection amount on this particular LC-MS/MS setup (dashed line).

resulted in the highest number of quantified proteins (Figure 3c, Figure S2, Table S1). Taken together, based on numbers of identified and quantified proteins, a rather broad window ranging from approximately 1.49 to $5.20 \mu \mathrm{g}$ of peptides was found as an optimal peptide injection amount for our LC-MS/MS setup.

In an attempt to further narrow down this window, we decided to evaluate selected LC and MS parameters. The efficient time consumption of a Q-Exactive HF mass spectrometer is, among other things, reflected by the ion injection time. The closer to ideal the amount of material presented to the mass spectrometer is, the lower the injection time will be. Figure 4a (left panel) shows that the average MS2 ion injection time decreases with increasing peptide amount, leveling off and reaching an optimal minimum around an average amount of $3.28 \mu \mathrm{g}$ of injected peptides. We also assessed the signal-to-noise $(\mathrm{S} / \mathrm{N})$ ratio of chromatographic peaks and peak areas, two LC parameters that were extracted for all identified peptides using the moFF algorithm (Argentini et al., 2016). Figure 4a (right panel) shows that the average $\mathrm{S} / \mathrm{N}$ ratio increases with increasing peptide amounts and again levels off at an average amount of about $3.28 \mu \mathrm{g}$ of injected peptides. Analysis of the median peak area of all identified peptides showed a maximum between 5.20 and $7.08 \mu \mathrm{g}$ for the two replicate experiments (Figure 4a (middle)). When overloading a reversed phase $\mathrm{C}_{1} 8$ column, one expects peak tailing, with a concomitant increase of the average peak area due to repulsion effects of same-charge ions (Johnson et al., 2013; McCalley, 2003, 2010). The observed leveling off and even decrease of the peak area at higher loads though contradicted this expectation. To explore this further, we split peptides according to their retention time interval into three parts: early eluting peptides (hydrophilic, $n=126$ ), late eluting peptides (hydrophobic, $\mathrm{n}=178$ ) and the ones eluting in between (mid eluting/intermediate hydrophobic peptides, n=2304) (Figure S1). This classification revealed an interesting trend especially for the hydrophilic peptides, consisting of a considerable decline in peak area starting from around 2-3 $\mu \mathrm{g}$ 
bioRxiv preprint doi: https://doi.org/10.1101/703397; this version posted July 16,2019 . The copyright holder for this preprint (which was not certified by peer review) is the author/funder, who has granted bioRxiv a license to display the preprint in perpetuity. It is made available under aCC-BY-NC-ND 4.0 International license.

a
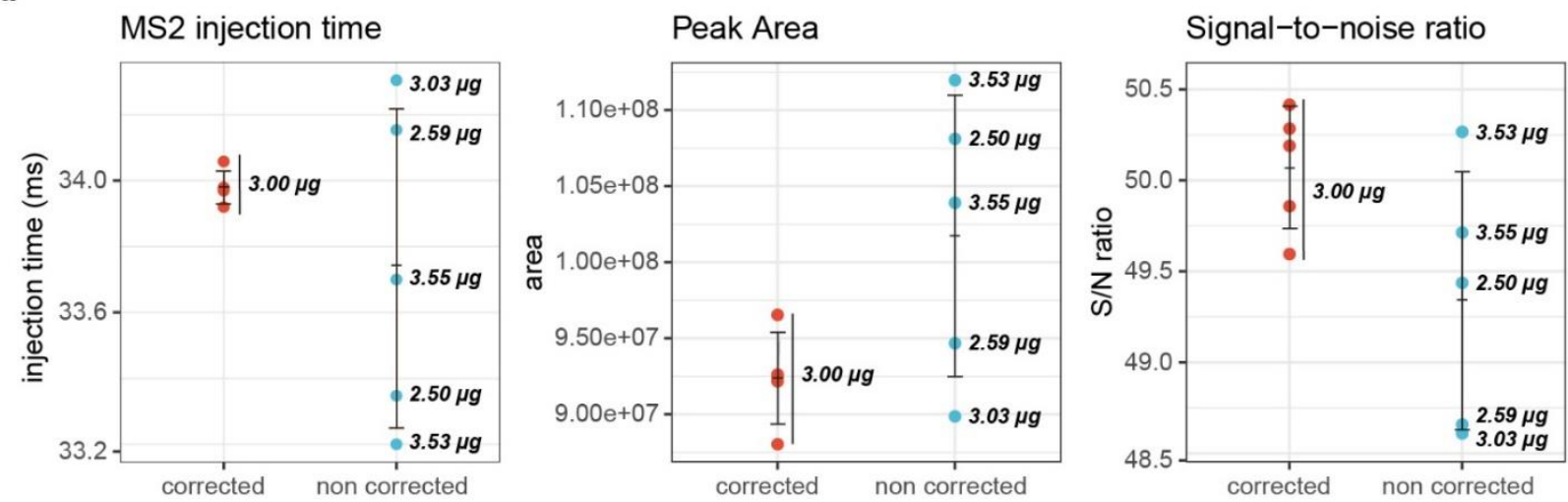

b
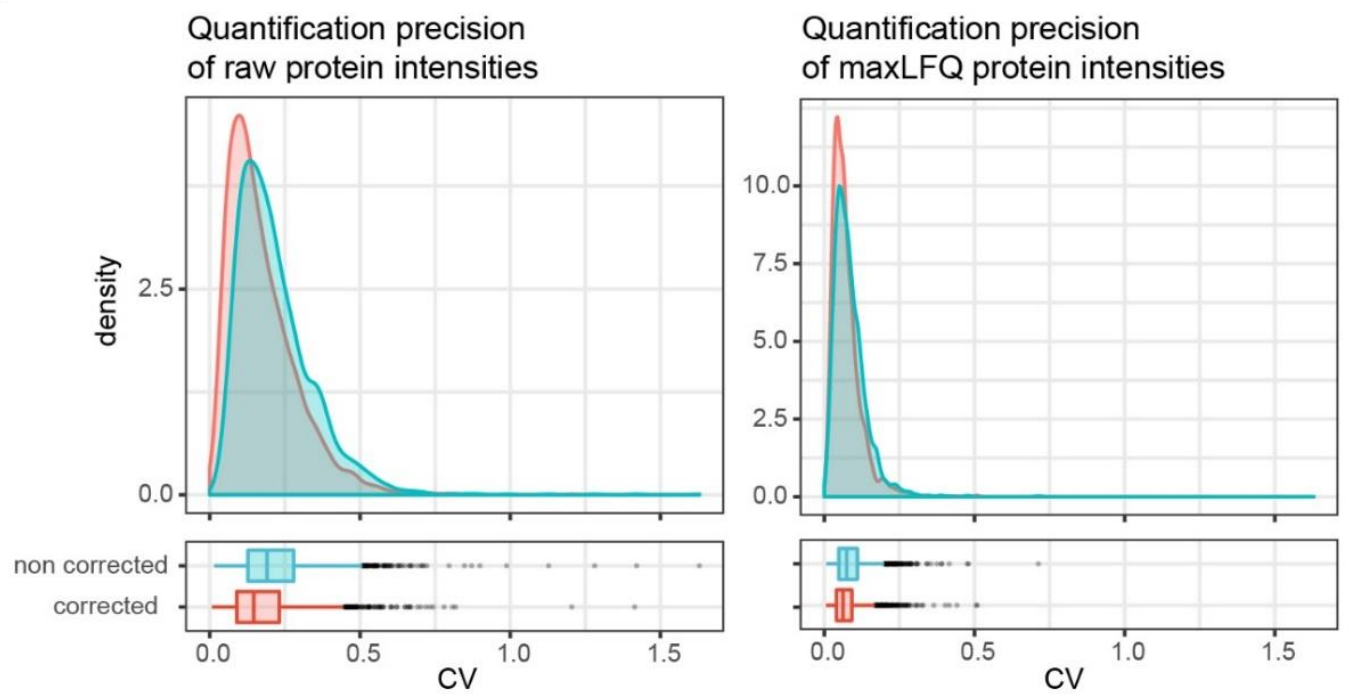

Figure 5- Comparison of LC-MS/MS and protein quantification reproducibility for two sets of analytical runs on trypsin digested Hek293T cell extracts. (a) Plots of the average MS2 injection time, median peak area and median signal-to-noise ratio values are shown for the identified peptides in each of five replicates from the 'corrected' and 'non corrected' groups of samples. For each data point, the exact injected peptide amount based on Lunatic quantification is shown on the right. Vertical bars represent mean \pm standard deviation. (b) Density plots and boxplots showing the distribution of the coefficients of variation (CV) of raw intensities (left) and (normalized) maxLFQ intensities (right) for all proteins quantified in the 10 analytical runs ( $\mathrm{n}=2677$ ). For both raw and normalized protein intensities, a shift to higher CV values was found for the 'non-corrected' samples (Mann-Whitney U test pvalue $<2.2 \mathrm{X1O}^{-16}$ for the shift in both raw and normalized distributions).

for both replicate series (Figure 4 b), pointing to a loss of these peptides.

This loss is most likely related to the use of a trapping column in our LC-MS/MS setup, whose capacity appears not to allow retention of the most hydrophilic peptides for higher sample amounts. In contrast, the hydrophobic peptides, having a higher affinity for the stationary phase, do not elute from the trapping column during the washing step that precedes the analytical column chromatography. As a consequence, the more peptides were loaded, the more the peak area of hydrophobic peptides would increase, as shown in Figure $4 \mathrm{~b}$ (right panel). The peptides belonging to the middle region do not have a pronounced affinity for the stationary phase, and will therefore have a mixed behavior (Figure 4b, middle panel). The disadvantage of losing hydrophilic peptides does not overrule the advantages of using a trapping column though, if void volumes are avoided (Mitulović et al., 2003). Therefore, based on the loss of hydrophilic peptides for amounts above $2.86 \mu \mathrm{g}$ and $2.63 \mu \mathrm{g}$ for replicate series 1 and 2 , respectively (peptide amounts that correspond to the peak maxima in the hydrophilic peptides peak area plot), together with the stabilization of the other parameters also around these sample amounts, we now routinely use $3.0 \mu \mathrm{g}$ of peptides as an optimal injection amount on this particular LC-MS/MS setup.

By running a series of LC-MS/MS analysis, covering a range of known peptide sample amounts, and performing a follow-up evaluation of the numbers of identified and 
quantified features, as well as of a few LC and MS parameters, we were able to identify $3 \mu \mathrm{g}$ as an optimal amount of injected peptides for our LC-MS/MS setup. This procedure could be applied to any other system and as such be of great use in proteomics laboratories. Indeed, knowledge about the most adequate sample amounts to use is currently not known for most systems. As a workaround to deal with this lack of information, it is common to, either inject different concentrations of the same sample, or else to perform short off-line analytical runs to fine-tune sample loading for a given sample (Pynn, Christopher Samonig, Krssakova, Mechtler, Decrop, \& Swart, n.d.). However, these strategies are time and cost ineffective and often consume higher amounts of precious sample material than the simple UV-based procedure described here.

Control over peptide amount injections effectively stabilizes LC-MS/MS and protein quantitation reproducibility

During sample preparation, it is common to make the protein amounts for all samples of a batch even before proceeding to the digestion step. This will generate very equivalent MS samples that can then be injected for LC-MS/MS using equal volumes. We decided to evaluate if, in comparison to that approach, performing peptide quantification prior to MS and concomitantly adjusting injection volumes to account for small differences in samples concentrations would have an impact on the reproducibility of LC-MS/MS and, in particular, of quantitation data.

To this end, we analyzed five tryptic digests of HEK293T cells, prepared in parallel starting from five different cell pellets. On a first set of analytical runs, we corrected for the peptide amounts loaded based on the Lunatic concentration, having injected exactly $3.0 \mu$ g of peptide mixture for all five replicates. In contrast, on a second set of runs of the same samples, we injected a fixed volume, corresponding to the average used for the first experiment. After calculating the peptide loadings for this second experiment, we realized there was a difference of $1 \mu \mathrm{g}$ between the lowest and highest concentration sample $(2.5 \mu \mathrm{g}$ and $3.55 \mu \mathrm{g}$, respectively). These differences are quite substantial and show the importance of accurate peptide quantitation of MS-ready samples. Even with automation of sample preparation, which should alleviate heterogeneity between samples, peptide quantitation could always be kept as a quality check step.

For both sets, the previously used MS and LC parameters were examined (Figure 5a). Strikingly, while all MS injection time, peak area and $\mathrm{S} / \mathrm{N}$ noise ratio values were close to each other in the experiment with the "corrected" sample amounts, these showed a much higher spread in the second experiment where each analytical run had a different sample amount. Indeed, the standard deviation for MS2 injection time, peak area and $\mathrm{S} / \mathrm{N}$ ratio had a 10-fold, a 3-fold and a 2-fold increase, respectively, for the 'non-corrected' experiment relative to the 'corrected' experiment (Figure 5a).

Finally, we checked if this higher analytical reproducibility also resulted in a more precise protein quantification. To this end, we looked at the distribution of the coefficients of variation $(\mathrm{CV})$ of raw protein intensity values from corrected replicates versus that from non-corrected replicates. In addition, we compared the $\mathrm{CV}$ distributions for LFQ protein intensity values obtained after normalization by the MaxLFQ algorithm. As expected, CV values for the non-normalized intensities were significantly lower for the 'corrected' experiment (Figure 5b, Mann-Whitney U test $\mathrm{p}$-value $<2.2 \mathrm{x}^{-16}$ ). However, a shift towards lower $\mathrm{CV}$ values was also observed for the normalized LFQ intensities of the "corrected" experiment (Mann-Whitney U test $\mathrm{p}$-value $<2.2 \mathrm{x1O}^{-16}$ ), indicating that computational normalization cannot fully compensate for differences in injection amounts. Such reduction in the dispersion of protein quantification estimates can potentially improve the sensitivity for detecting peptide and protein regulation. Together, these data show that working consistently with equal peptide amounts results in increased reproducibility LCMS/MS and protein quantification data, even in combination with normalization of protein intensities during data analysis.

\section{Conclusion}

In the present work, we describe a simple procedure to quantify MS-ready peptides using the Lunatic microfluidic UV-visible spectrophotometer from Unchained Labs. The Lunatic instrument, with its integrated peptide quantification software application, constitutes an accurate and easyto-use device for the implementation of peptide quantification in proteomics laboratories and facilities. Through careful control of the injected peptide amount on a commonly used LC-MS/MS setup, we show how this procedure can provide a constant quality boost to MS-based proteome analyses. Application of this methodology on a routine basis can drastically reduce time and sample losses by avoiding re-runs after injection of suboptimal peptide amounts.

\section{ASSOCIATED CONTENT}

\section{Supporting Information.}

Figure $S_{1}$ - Schematic plot showing the percent solvent B gradient and total ion current along retention time from a representative LC-MS/MS run of the sample amount optimization experiment. Retention time points used for peptide partioning are also shown.

Figure S2 - Heatmap visualizing the intensities of quantified proteins in each of the 10 analytical runs measured using LC column 2. Missing values are shown in gray.

Table $S_{1}$ - Numbers of identified and quantified peptide and protein groups for the two replicate series of $\mathrm{K}_{5} 62$ analytical runs. 
bioRxiv preprint doi: https://doi.org/10.1101/703397; this version posted July 16,2019 . The copyright holder for this preprint (which was not certified by peer review) is the author/funder, who has granted bioRxiv a license to display the preprint in perpetuity. It is made available under aCC-BY-NC-ND 4.0 International license.

This material is available free of charge via the Internet at http://pubs.acs.org.

\section{AUTHOR INFORMATION}

\section{Corresponding Authors}

* E-mail: francis.impens@vib-ugent.be

*E-mail: kris.gevaert@vib-ugent.be

\section{Author Contributions}

K.P., T.M., F.I., K.G., A.S and J.P. conceptualized the study and designed the experiments. K.P. and T.M.M. prepared the yeast and $\mathrm{K}_{5} 62$ cell peptide dilution samples. K.B prepared the Hek293T proteome digests. J.P. performed the LCMS/MS measurements. T.M., A.S, A.A and J.P. analyzed the data. T.M wrote the manuscript with input from F.I., K.G., L.M. All authors revised the final manuscript.

\section{Funding Sources}

The authors would like to acknowledge the VIB Tech Watch Fund for supporting early access to innovative technology platforms. L.M., K.G. and F.I. acknowledge support by the EPIC-XS consortium and F.I. acknowledges support by FWOSBO Project Soo6617N.

\section{Notes}

The authors declare no competing financial interest.

\section{ACKNOWLEDGMENT}

The authors thank Lisa Adamiak and Dina Finan from Unchained Labs for technical advice and sharing information about the Lunatic instrument specifications.

\section{ABBREVIATIONS}

LC-MS/MS, liquid chromatography-tandem mass spectrometry; LFQ, label free quantification.

\section{REFERENCES}

Argentini, A., Goeminne, L. J. E., Verheggen, K., Hulstaert, N., Staes, A., Clement, L., \& Martens, L. (2016). Using moFF to Extract Peptide Ion Intensities from LC-MS experiments. Retrieved from http://dx.doi.org/10.1038/protex.2016.085

Bache, N., Geyer, P. E., Bekker-Jensen, D. B., Hoerning, O., Falkenby, L., Treit, P. V, ... Mann, M. (2018). A Novel LC System Embeds Analytes in Pre-formed Gradients for Rapid, Ultra-robust Proteomics. Molecular \& Cellular Proteomics: MCP, 17(11), 2284-2296. https://doi.org/10.1074/mcp.TIR118.00o853

Batth, T. S., Tollenaere, M. A. X., Rüther, P., Gonzalez-Franquesa, A., Prabhakar, B. S., Bekker-Jensen, S., ... Olsen, J. V. (2019). Protein Aggregation Capture on Microparticles Enables Multipurpose Proteomics Sample Preparation. Molecular \& Cellular Proteomics: MCP, 18(5), 1027-1035. https://doi.org/10.1074/mcp.TIR118.001270

Bittremieux, W., Tabb, D. L., Impens, F., Staes, A., Timmerman, E., Martens, L., \& Laukens, K. (2017). Quality control in mass spectrometry-based proteomics. Mass Spectrometry Reviews, (March), 1-15. https://doi.org/10.1002/mas.21544

Bittremieux, W., Valkenborg, D., Martens, L., \& Laukens, K. (2017). Computational quality control tools for mass spectrometry proteomics. Proteomics, 17(3-4), 3-4. https://doi.org/10.1002/pmic.201600159

Bittremieux, W., Walzer, M., Tenzer, S., Zhu, W., Salek, R. M., Eisenacher, M., \& Tabb, D. L. (2017). The Human Proteome Organization-Proteomics Standards Initiative Quality Control
Working Group: Making Quality Control More Accessible for Biological Mass Spectrometry. Analytical Chemistry, 89(8), 4474-4479. https://doi.org/10.1021/acs.analchem.6bo4310

Chiva, C., Olivella, R., Borràs, E., Espadas, G., Pastor, O., Solé, A., \& Sabidó, E. (2018). QCloud: A cloud-based quality control system for mass spectrometry-based proteomics laboratories. PLoS ONE, 13(1), e0189209. https://doi.org/10.1371/journal.pone.o189209

Cox, J., Hein, M. Y., Luber, C. A., Paron, I., Nagaraj, N., \& Mann, M. (2014). Accurate Proteome-wide Label-free Quantification by Delayed Normalization and Maximal Peptide Ratio Extraction, Termed MaxLFQ. Molecular \& Cellular Proteomics, 13(9), 25132526. https://doi.org/10.1074/mcp.M113.031591

Cox, J., \& Mann, M. (2008). MaxQuant enables high peptide identification rates, individualized p.p.b.-range mass accuracies and proteome-wide protein quantification. Nature Biotechnology, 26, $1367 . \quad$ Retrieved from http://dx.doi.org/10.1038/nbt.1511

D. Kelstrup, C., B. Bekker-Jensen, D., N. Arrey, T., Hogrebe, A., Harder, A., \& V. Olsen, J. (2017). Performance Evaluation of the Q Exactive HF-X for Shotgun Proteomics. Journal of Proteome Research, $\quad 17(1), \quad 727-738$. https://doi.org/10.1021/acs.jproteome.7boo6o2

Espadas, G., Borràs, E., Chiva, C., \& Sabidó, E. (2017). Evaluation of different peptide fragmentation types and mass analyzers in data-dependent methods using an Orbitrap Fusion Lumos Tribrid mass spectrometer. Proteomics, 17(9), 1-9. https://doi.org/10.1002/pmic.201600416

Fu, Q., Kowalski, M. P., Mastali, M., Parker, S. J., Sobhani, K., van den Broek, I., ... Van Eyk, J. E. (2018). Highly Reproducible Automated Proteomics Sample Preparation Workflow for Quantitative Mass Spectrometry. Journal of Proteome Research, $\quad 17(1), \quad 420-428$. https://doi.org/10.1021/acs.jproteome.7boo623

Guryča, V., Roeder, D., Piraino, P., Lamerz, J., Ducret, A., Langen, H., \& Cutler, P. (2014). Automated Sample Preparation Platform for Mass Spectrometry-Based Plasma Proteomics and Biomarker Discovery. Biology (Vol. https://doi.org/10.339o/biology3010205

Hinzke, T., Kouris, A., Hughes, R.-A., Strous, M., \& Kleiner, M. (2019). More Is Not Always Better: Evaluation of $1 \mathrm{D}$ and 2D-LC-MS/MS Methods for Metaproteomics. Frontiers in Microbiology, 10, 238. https://doi.org/10.3389/fmicb.2019.00238

Johnson, D., Boyes, B., \& Orlando, R. (2013). The Use of Ammonium Formate as a Mobile-Phase Modifier for LC-MS/MS Analysis of Tryptic Digests. Journal of Biomolecular Techniques :JBT, 24(4), 187-197. https://doi.org/10.7171/jbt.13-2404-005

Kulak, N. A., Geyer, P. E., \& Mann, M. (2017). Loss-less Nanofractionator for High Sensitivity, High Coverage Proteomics. Molecular \& Cellular Proteomics, 16(4), 694-705. https://doi.org/10.1074/mcp.O116.065136

Kulak, N. A., Pichler, G., Paron, I., Nagaraj, N., \& Mann, M. (2014). Minimal, encapsulated proteomic-sample processing applied to copy-number estimation in eukaryotic cells. Nature Methods, 11(3), 319-324. https://doi.org/10.1038/nmeth.2834

McCalley, D. V. (2003). Rationalization of retention and overloading behavior of basic compounds in reversed-phase HPLC using low ionic strength buffers suitable for mass spectrometric detection. Analytical Chemistry, 75(14), 3404-3410.

McCalley, D. V. (2010). The challenges of the analysis of basic compounds by high performance liquid chromatography: Some possible approaches for improved separations. Journal of Chromatography A, $1217(6), \quad 858-880$. https://doi.org/https://doi.org/10.1016/j.chroma.2009.11.068

Meier, F., Brunner, A.-D., Koch, S., Koch, H., Lubeck, M., Krause, M., ... Mann, M. (2018). Online Parallel Accumulation-Serial Fragmentation (PASEF) with a Novel Trapped Ion Mobility Mass Spectrometer. Molecular \& Cellular Proteomics : MCP, 
bioRxiv preprint doi: https://doi.org/10.1101/703397; this version posted July 16, 2019. The copyright holder for this preprint (which was not certified by peer review) is the author/funder, who has granted bioRxiv a license to display the preprint in perpetuity. It is made available under aCC-BY-NC-ND 4.0 International license.

17(12), 2534-2545. https://doi.org/10.1074/mcp.TIR118.00o9oo

Mitulović, G., Smoluch, M., Chervet, J.-P., Steinmacher, I., Kungl, A., \& Mechtler, K. (2003). An improved method for tracking and reducing the void volume in nano HPLC-MS with micro trapping columns. Analytical and Bioanalytical Chemistry, 376(7), 946-951. https://doi.org/10.1007/soo216-003-2047-2

Moggridge, S., Sorensen, P. H., Morin, G. B., \& Hughes, C. S. (2018). Extending the Compatibility of the $\mathrm{SP}_{3}$ Paramagnetic Bead Processing Approach for Proteomics. Journal of Proteome Research, 17(4), 1730-1740. https://doi.org/10.1021/acs.jproteome.7boog13

Peng, J., Tang, F., Zhou, R., Xie, X., Li, S., Xie, F., ... Mu, L. (2016). New techniques of on-line biological sample processing and their application in the field of biopharmaceutical analysis. Acta $\begin{array}{lll}\text { Pharmaceutica Sinica 6(6), 540-551. } & \text { B, }\end{array}$ https://doi.org/10.1016/J.APSB.2016.05.016

Pynn, Christopher Samonig, M., Krssakova, G., Mechtler, K., Decrop, W., \& Swart, R. (n.d.). Capillary-flow LC-UV sample quality control for low-flow LC-MS based proteomics. Retrieved from https://assets.thermofisher.com/TFS-Assets/CMD/TechnicalNotes/tn-72602-lc-uv-ms-low-flow-proteomics-tn726o2en.pdf

Rieux, L., Lubda, D., Niederländer, H. A. G., Verpoorte, E., \& Bischoff, R. (2006). Fast, high-efficiency peptide separations on a $50-\mu \mathrm{m}$ reversed-phase silica monolith in a nanoLC-MS set-up. Journal of Chromatography A, 1120(1-2), 165-172. https://doi.org/10.1016/J.CHROMA.2006.01.038
Rieux, L., Sneekes, E.-J., \& Swart, R. (n.d.). How Sample Loadability of the NanoLC Column Influences Protein Identification in LCMS Proteomics Studies. Retrieved from https://assets.thermofisher.com/TFS-

Assets/CMD/posters/PO-70145-Sample-Loadability-ProteinIdentification-PO70145-EN.pdf

Rudnick, P. A., Clauser, K. R., Kilpatrick, L. E., Tchekhovskoi, D. V., Neta, P., Blonder, N., ... Stein, S. E. (2010). Performance Metrics for Liquid Chromatography-Tandem Mass Spectrometry Systems in Proteomics Analyses. Molecular \& Cellular Proteomics, 9(2), 225-241. https://doi.org/10.1074/mcp.M900223-MCP200

Tabb, D. L. (2013). Quality assessment for clinical proteomics. Clinical Biochemistry, 46(6), 411-420. https://doi.org/10.1016/J.CLINBIOCHEM.2012.12.003

Wiśniewski, J. R., \& Gaugaz, F. Z. (2015). Fast and Sensitive Total Protein and Peptide Assays for Proteomic Analysis. Analytical Chemistry, 87(8), 4110-4116. https://doi.org/10.1021/ac504689z

Zhu, Y., Piehowski, P. D., Zhao, R., Chen, J., Shen, Y., Moore, R. J., ... Kelly, R. T. (2018). Nanodroplet processing platform for deep and quantitative proteome profiling of 10-10o mammalian cells. Nature $\quad$ Communications, $\quad 9(1), \quad 882$. https://doi.org/10.1038/s41467-018-03367-w 
bioRxiv preprint doi: https://doi.org/10.1101/703397; this version posted July 16,2019 . The copyright holder for this preprint (which was not certified by peer review) is the author/funder, who has granted bioRxiv a license to display the preprint in perpetuity. It is made available under aCC-BY-NC-ND 4.0 International license.

Peptide quantification of MS-ready samples on UV-visible spectrophotometer

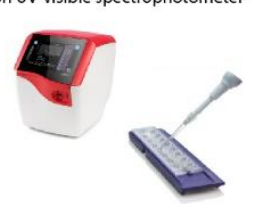

TIC plot

LC-MS-MS

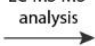

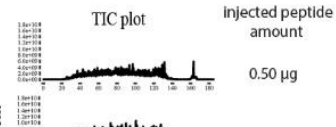

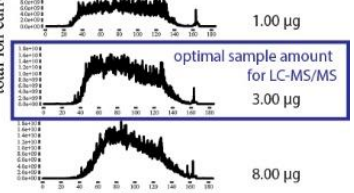

retention time [min] 